\title{
Single-Cell Microfluidics to Study the Effects of Genome Deletion on Bacterial Growth Behavior
}

\author{
Xiaofei Yuan, ${ }^{\dagger}$ Jillian M. Couto, ${ }^{\ddagger}$ Andrew Glidle, ${ }^{\dagger}$ Yanqing Song, ${ }^{\dagger}$ William Sloan, ${ }^{*}$,
} and Huabing Yin*, ${ }^{+}$

${ }^{\dagger}$ College of Science and Engineering, Division of Biomedical Engineering, School of Engineering, University of Glasgow, Glasgow G12 8QQ U.K.

\#College of Science and Engineering, Division of Infrastructure and Environment, School of Engineering, University of Glasgow, Glasgow G12 8QQ U.K.

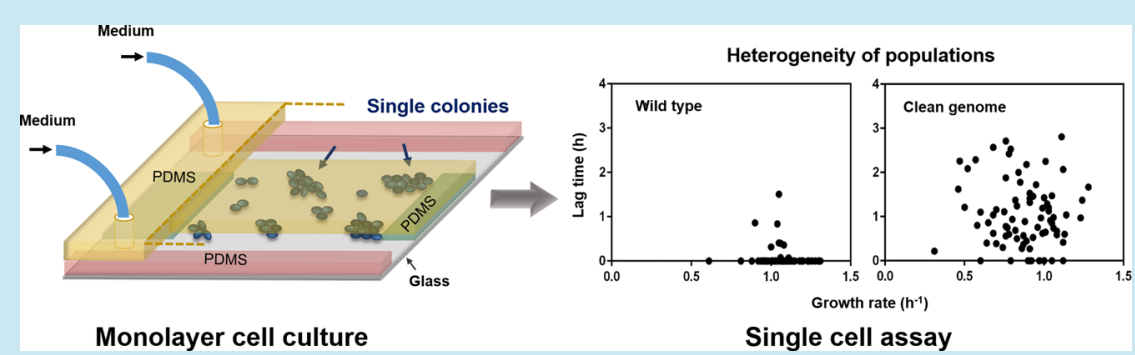

ABSTRACT: By directly monitoring single cell growth in a microfluidic platform, we interrogated genome-deletion effects in Escherichia coli strains. We compared the growth dynamics of a wild type strain with a clean genome strain, and their derived mutants at the single-cell level. A decreased average growth rate and extended average lag time were found for the clean genome strain, compared to those of the wild type strain. Direct correlation between the growth rate and lag time of individual cells showed that the clean genome population was more heterogeneous. Cell culturability (the ratio of growing cells to the sum of growing and nongrowing cells) of the clean genome population was also lower. Interestingly, after the random mutations induced by a glucose starvation treatment, for the clean genome population mutants that had survived the competition of chemostat culture, each parameter markedly improved (i.e., the average growth rate and cell culturability increased, and the lag time and heterogeneity decreased). However, this effect was not seen in the wild type strain; the wild type mutants cultured in a chemostat retained a high diversity of growth phenotypes. These results suggest that quasi-essential genes that were deleted in the clean genome might be required to retain a diversity of growth characteristics at the individual cell level under environmental stress. These observations highlight that single-cell microfluidics can reveal subtle individual cellular responses, enabling in-depth understanding of the population.

KEYWORDS: single-cell, microfluidics, cell growth, genome reduction, mutation

ynthetic biology has adopted the idea of chassis organisms with reduced genomes that are engineered to efficiently express essential endogenous proteins. The reduced genomes of these chasses present a stable and predictable platform upon which synthetic genes and circuits can be rationally designed and then embedded. ${ }^{1-4}$ The development of a chassis genome involves identifying a set of essential genes, usually via sequence alignment with strains of the same species, and then deleting anything deemed nonessential. ${ }^{1}$ This should theoretically have the additional advantage of reducing the associated energy cost (energy per gene) of replicating a larger genome and expressing genes that are not essential for the desired environment (protein burden). ${ }^{5}$ However, rather paradoxically, genome reductions appear to slow growth rates. ${ }^{2,6}$ This has been taken as evidence for a reduction in fitness and has led to the suggestion that in addition to essential genes, genomes might also contain quasi-essential genes that function to promote fast growth. ${ }^{2}$
We know that even in supposedly clonal populations of bacteria there can be unexplained "stochastic" variability in the phenotypical behavior of individual cells. ${ }^{7-9}$ Indeed, there is evidence from natural microbial communities that a substantial number of cells do not contribute to genetic diversity of subsequent generations, which suggests that not all cells contribute to growth events. This result has been inferred by estimating the effective population size. ${ }^{10}$ It is not known whether this stochastic variability in phenotype is increased or diminished in minimal genomes, because we typically observe growth at the population level rather than in individuals. Furthermore, synthetic organisms are usually tested in highly controlled laboratory conditions, so it is unclear if the heterogeneity in phenotype is exacerbated by environmental

Received: May 27, 2017

Published: August 26, 2017 
(A)
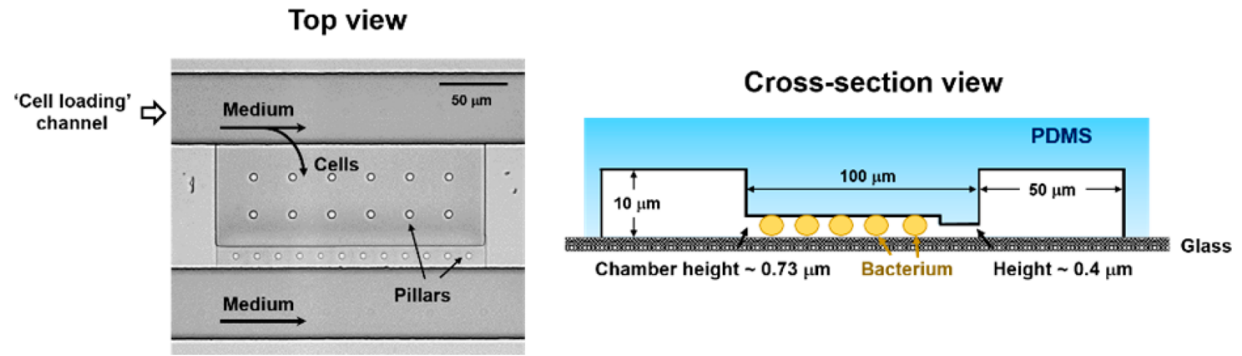

(B)

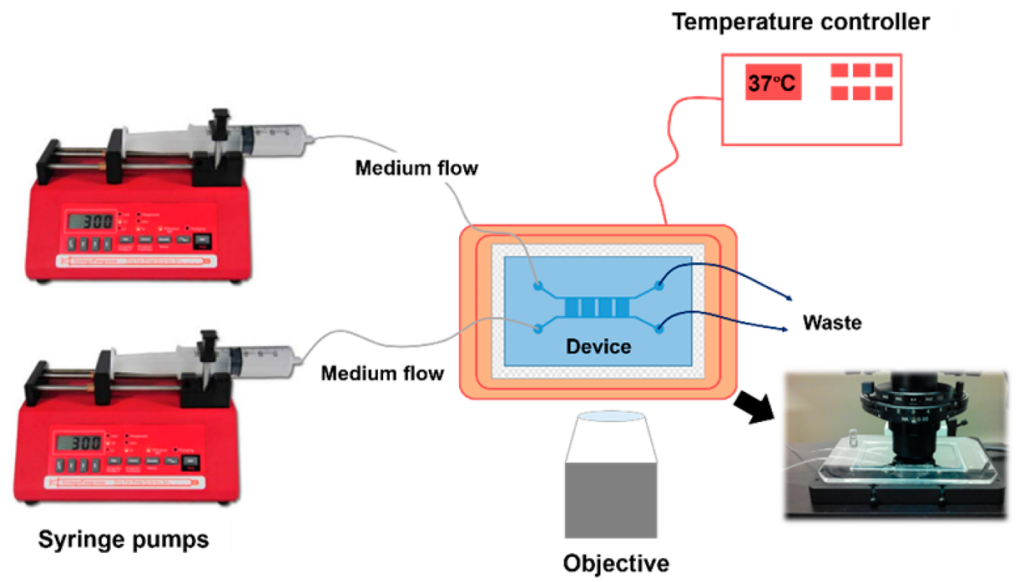

Figure 1. (A) The design of the microfluidic device. The side channels were used to deliver cells and medium. A monolayer of cells was trapped in the middle chamber. (B) Outline of the experiment setup.

stress. The chassis is fundamental to any synthetic biotechnology, therefore not only is it essential to work out the complexities of chassis gene components, it is also important to be able to reliably characterize growth and, where variability occurs, potentially select fractions of the population that offer the highest growth and the least variability.

Microfluidics provides an ideal platform to culture and monitor individual bacterial cells over many generations. ${ }^{1-16}$ It allows multiparameter, near-continuous measurements of dividing cells in high throughput devices with a bewildering range of spatial architectures and with low sample consumption. ${ }^{17,18}$ Recently, we have developed a simple gradient microfluidic system for a rapid bacterial growth inhibition test at the single-cell level. ${ }^{19}$ With this approach, quantitative and dynamic growth information about individual cells within a population can be obtained in parallel under a wide range of conditions. Importantly, it provided a powerful platform to mimic naturally occurring habitats of environmental microbes, enabling a close-to-reality analysis of bacterial responses to stresses and consequently the discovery of new phenomena. ${ }^{20}$

In this work, we have exploited single-cell microfluidics to investigate the influence of genome reduction on microbial growth, aiming to reveal the diversity of individual behaviors to gain an in-depth understanding of the population. For this purpose, the lab reference strain Escherichia coli, K12 MG1655 (denoted as the wild type, WT), and the derived genomereduced strain, MDS42 (denoted as the clean genome, CG), were used. This CG strain is formed by deleting $14.3 \%$ of the WT's genome, with the deleted sections including nonessential genes, mobile DNA elements and cryptic prophages. ${ }^{1}$ The CG strain has been reported to be a good chassis for synthetic biology applications because of plasmid stability, low mutation rate, and protein production. ${ }^{1,21-23}$ However, this low mutation rate appears to hold only when the $E$. coli are in optimal laboratory condition; Couto et al. ${ }^{24}$ showed that under glucose starvation stress, as described in Ferenci et al., ${ }^{25}$ mutation rates indexed in the $r p o B$ gene $\left(\mathrm{Rif}^{\mathrm{R}}\right)$ or $c y c A$ gene $\left(c y c^{\mathrm{R}}\right)$, were orders of magnitude higher in both the WT and CG organisms. Most mutations correlated with a decrease in growth rate and ultimately failed to gain a foothold in the population. A few, however, appeared to remain fit enough to be retained in the population over time. However, a comparison of the diversity in growth characteristics of the WT and CG mutants that can survive these harsh conditions has not been made. Thus, here, we cultured mutant subpopulations in oligotrophic conditions, minimal media ${ }^{26}$ supplemented with $1 \mathrm{mM}$ glucose in a chemostat with a dilution rate of $0.1 \mathrm{~h}^{-1}$. This dilution rate meant that only mutants capable of growing at a rate that was greater than or equal to $0.1 \mathrm{~h}^{-1}$ would be retained; the slower growers would be washed out. As described below, using microfluidics allows us to rapidly characterize the diversity in growth characteristics of the mutants that could survive.

To enhance throughput and flexibility of cell handing, a new generation of microfluidic device was developed that consisted of an array of microchambers to allow monolayer cell culture and real-time monitoring of cell growth (Figure 1). Single-cell growth characteristics, such as specific growth rate $\left(\mu, \mathrm{h}^{-1}\right)$, lag time $(\lambda, h)$, and culturability, were quantified. This enables a direct correlation of growth rate and lag time of individual cells that reveals the population heterogeneity-information that is difficult to obtain using conventional well-plate approaches. For the first time, the effects of genome reduction and random mutation on population heterogeneity were measured, which indicate that the deleted genes are not as redundant as previously believed, but play an important role to resist stress and homogenize the population. 

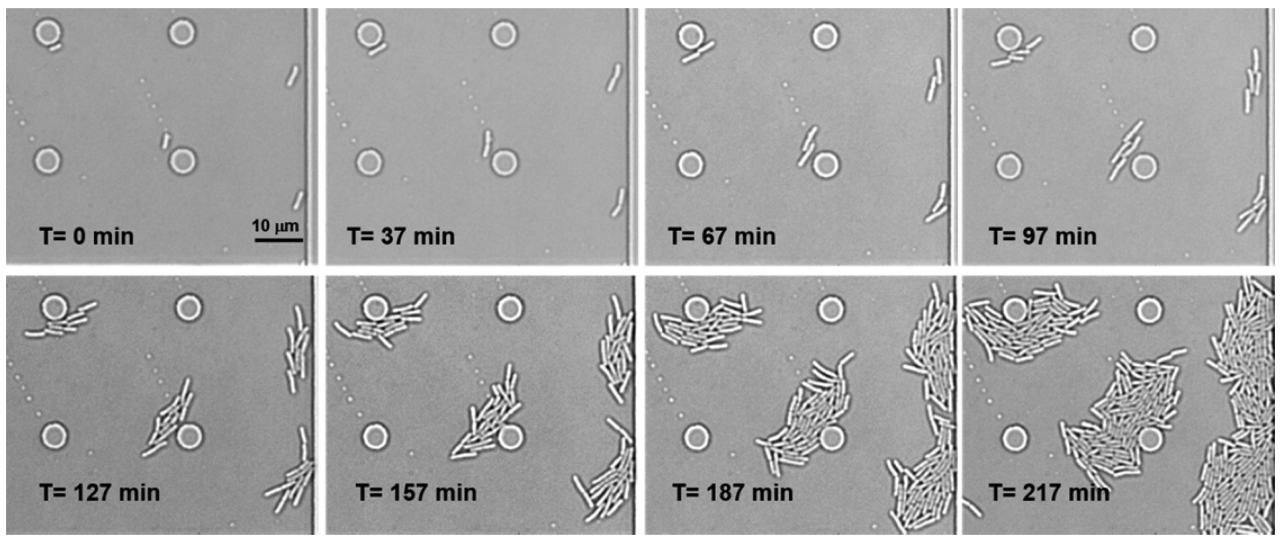

Figure 2. Typical time-lapse optical images of the WT strain in microchambers under a continuous medium flow. It should be noted that cells are randomly located in chambers.

(A)

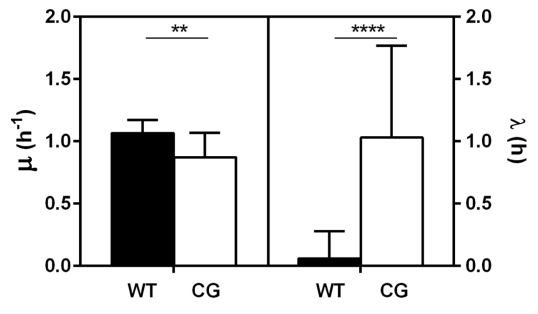

(B)

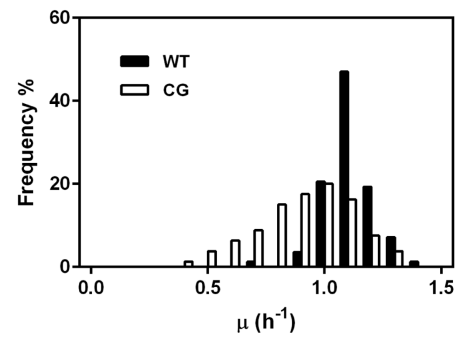

(C)

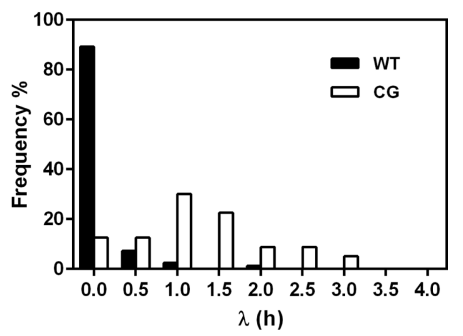

Figure 3. (A) Comparison of the average values for the specific growth rate $\mu$ and the lag time $\lambda$ of the WT and the CG strains ( $n=80$ for each strain). (B, C) Histogram distributions of $\mu$ and $\lambda$ of these two strains. $p<0.0001(* * * *) ; p<0.005(* *)$.

\section{RESULTS}

Effects of Genome Reduction on Cell Growth. Growth rates of both WT (i.e., MG1655) and CG (i.e., MDS42) strains have been previously shown to be unequal. ${ }^{6}$ We confirmed these findings via batch growth, where the specific growth rate $\mu$ is $1.04 \mathrm{~h}^{-1}$ (or doubling time of $40 \mathrm{~min}$ ) for the WT strain and $0.78 \mathrm{~h}^{-1}$ (or doubling time of $53 \mathrm{~min}$ ) for the CG strain (Supporting Information, Figure S1). However, since these measures represented average responses of the population, it is unclear what the main contributor to this discrepancy is. For instance, does the lower growth rate in the CG strain result from a reduced growth of all the cells or only from the slowest growing subset? In this context, microfluidics devices (Figure 1) were employed to characterize the growth kinetics of individual cells, revealing quantitative information on diverse behaviors within the populations.

Figure 2 illustrates typical time-lapse images of single cells as they gradually grew and divided to form microcolonies in the microchambers. From these images, growth curves for randomly selected single cells were obtained (Supporting Information, Figure S2). On-chip culture was under a continuous flow condition, which meant that after a short lag phase, exponential growth was observed and sustained for the duration of the experiment (because the substrates were continually replenished, Supporting Information, Figure S2). This enabled an accurate derivation of colony specific (individual) growth rate $(\mu)$ and lag time $(\lambda)$ parameters for a given substrate concentration as described in the Materials and Methods.

Figure 3 shows the distributions of such individual $\mu$ and $\lambda$ parameters from 80 randomly picked dividing cells. The average growth rates calculated using the on-chip single cell approach were consistent with the results from batch culture (Supporting Information, Figure S1), with the WT strain $(\mu=$ $\left.1.0 \mathrm{~h}^{-1}\right)$ growing significantly faster than the CG strain $(\mu=0.8$ $\left.\mathrm{h}^{-1}\right)(p<0.001)$ (Figure 3A). A histogram of the growth rate, $\mu$, of single cells showed that this slower average growth in the CG strain was a result of a majority of CG cells growing slower than those of the WT strain (Figure 3B). Approximately $80 \%$ of the WT cells had $\mu>1.0 \mathrm{~h}^{-1}$ in comparison to only $40 \%$ of the CG cells having $\mu>1.0 \mathrm{~h}^{-1}$ (Figure 3B). Furthermore, it was found $>90 \%$ of the WT cells showed no lag time (Figure 3C), whereas approximately $80 \%$ of the CG cells had a lag time between 0.5 and $3 \mathrm{~h}$. As a result, the mean lag time of the CG strain was significantly longer $(p<0.00001$, Figure 3A). It should be noted an extended lag time is indicative of the weak ability of a cell to acclimatize to a new environment (e.g., from broth suspension to the surface of a glass substrate), therefore indicating a negative effect of genome reduction on cell growth.

Effects of Starvation Induced Random Mutations. To investigate the impact of emerging genetic variation on the growth kinetics of these strains, we developed two populations of mutants, one from each strain. Each community was created by harvesting mutants from a glucose-limited chemostat that maintained a population at a dilution rate of $0.1 \mathrm{~h}^{-1}$ for 21 days. ${ }^{24}$ Glucose-limitation has been shown to promote random mutations in strains of $E$. coli $i^{25}$ that can be screened via acquired resistance to Rifampicin ${ }^{27}$ or Cycloserine, ${ }^{28}$ two commonly used antibiotics in lab evolution experiments. We exploited this system to produce organisms with mutations in either the $r p o B$ gene $\left(\right.$ rif $\left.^{\mathrm{R}}\right)$ or $c y c A$ gene $\left(\mathrm{cyc}^{\mathrm{R}}\right)$ in both the WT and CG strain backgrounds. Once harvested from the 
(A)
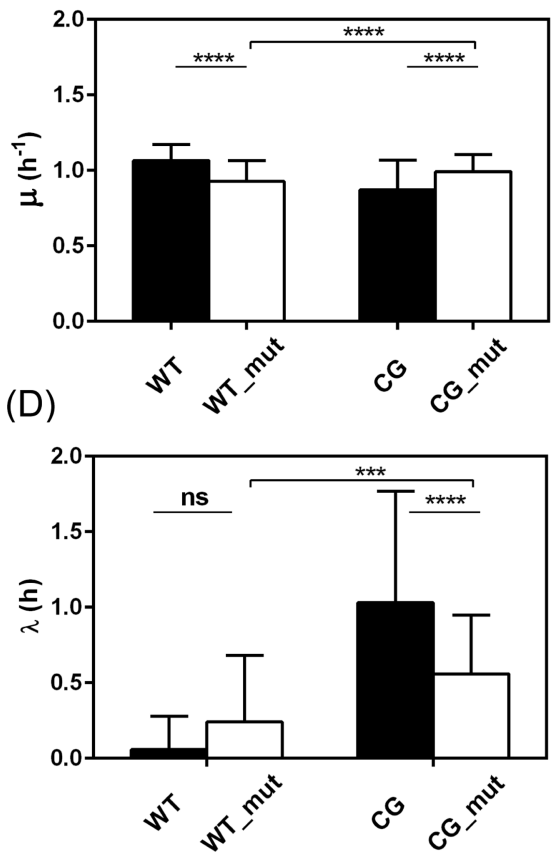

(B)

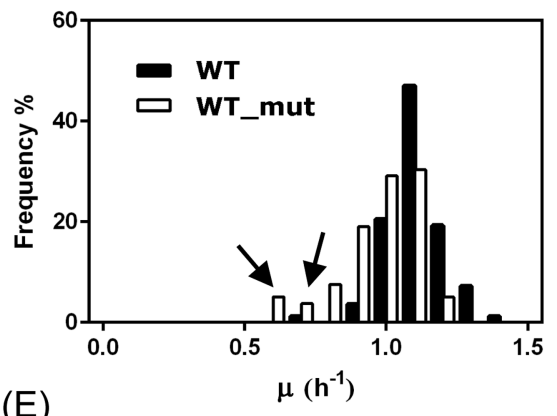

(E)

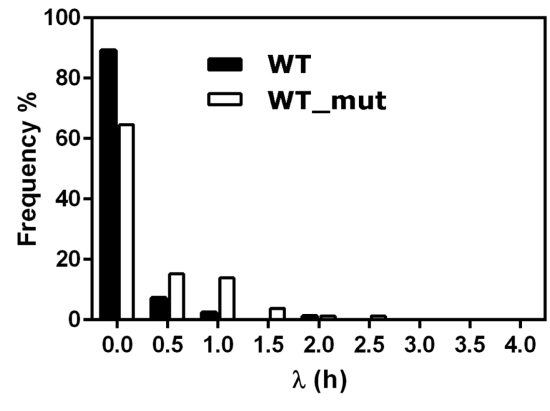

(C)

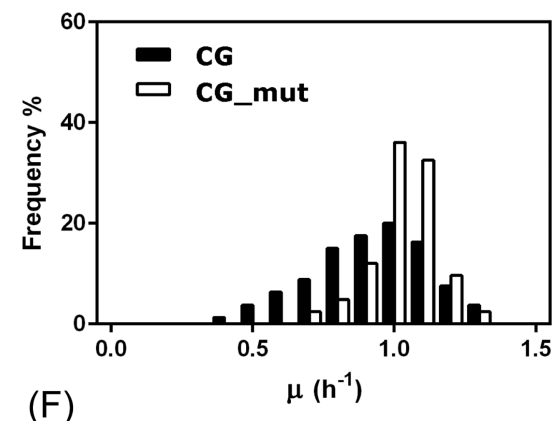

(F)

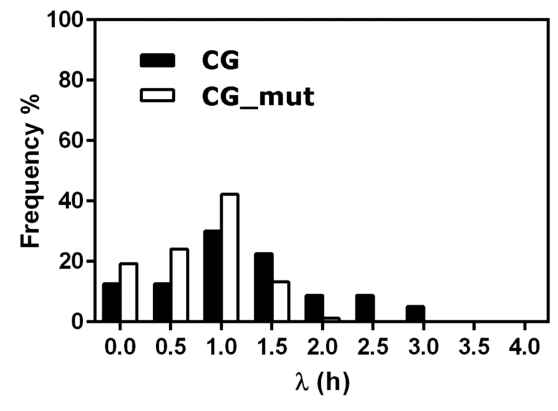

Figure 4. (A) Average values of the growth rates for the WT and CG strains, and the WT_mut and CG_mut populations together with (B, C) histograms of their distribution. (D) Average values of the lag times for the four populations together with (E, F) histograms of their distribution. Arrows in (B) indicate the subsets of slow growing cells. $p<0.0001(* * * *) ; p<0.0005(* * *)$; ns denotes not significant.

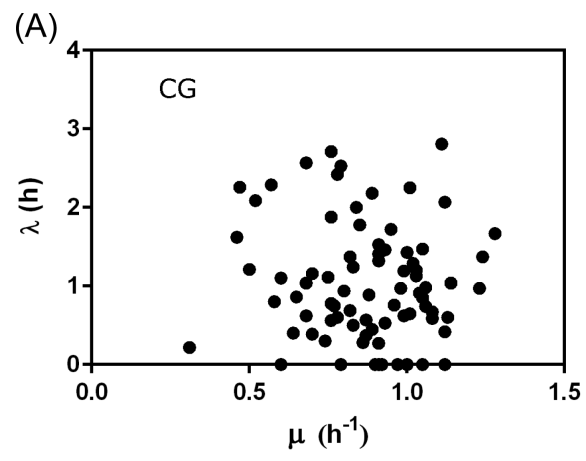

(C)

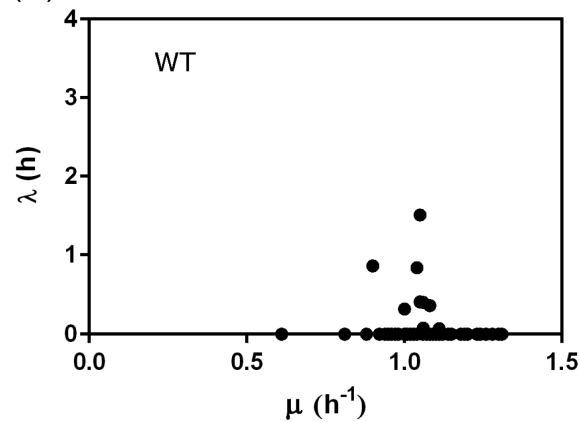

(B)

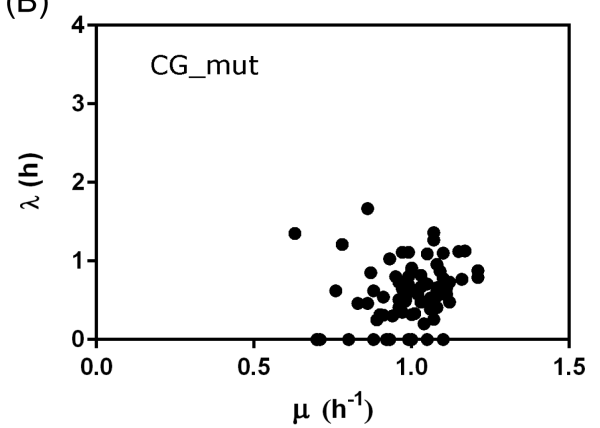

(D)

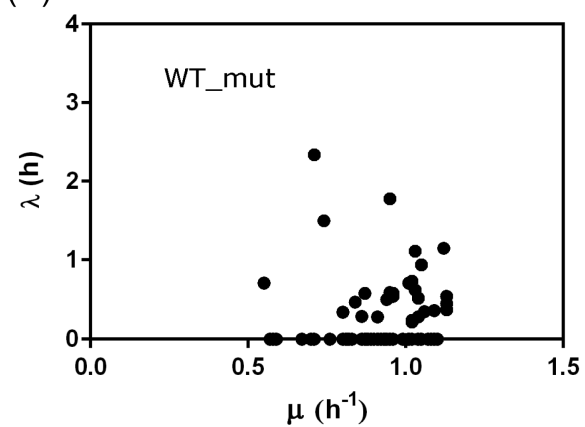

Figure 5. Scatter diagrams of the lag time $\lambda$ versus the specific growth rate $\mu$ of individual cells for the four populations. Each spot denotes a single cell.

chemostats, mutants from each strain background were designated WT_mut (mutants in the WT background) and CG_mut (mutants in the CG background). We started two new glucose-limited chemostat runs to grow up and maintain each of these communities of mutants, in the absence of any nonmutants. The chemostats were seeded with a subsample from each of the exponentially growing mutant communities, and operated with a dilution rate $0.1 \mathrm{~h}^{-1}$ for a period of $20 \mathrm{~h}$, by which time the density of organisms had reached a steady state $\left(\mathrm{OD}_{600}=0.1\right)$. Then samples of such cultures were taken for loading into the microfluidic devices.

As shown in Figure 4, the starvation induced random mutations generated contrasting growth effects for the WT and CG strains. The population of WT_mut cells showed a slightly 
Table 1. Average $\mu$ and $\lambda$ Values (Mean \pm Standard Deviation) of the Four E. coli Strains from On-Chip Culture

\begin{tabular}{|c|c|c|c|c|c|c|c|}
\hline \multicolumn{4}{|c|}{$\mu\left(\mathrm{h}^{-1}\right)$} & \multicolumn{4}{|c|}{$\lambda(\mathrm{h})$} \\
\hline WT & WT_mut & CG & CG_mut & WT & WT_mut & CG & CG_mut \\
\hline $1.07 \pm 0.10$ & $0.93 \pm 0.14$ & $0.87 \pm 0.19$ & $0.99 \pm 0.11$ & $0.06 \pm 0.22$ & $0.24 \pm 0.44$ & $1.03 \pm 0.74$ & $0.56 \pm 0.39$ \\
\hline
\end{tabular}

decreased average growth rate $\left(0.93 \pm 0.14 \mathrm{~h}^{-1}\right)$ but larger standard deviation in comparison to its parent WT strain $(1.07$ $\left.\pm 0.11 \mathrm{~h}^{-1}\right)(p<0.0001)$ (Figure $\left.4 \mathrm{~A}\right)$. The diversity of growth rates in the mutants and the presence of the slower growing mutants (Figure 4B, indicated by the arrows) is perhaps surprising, given that they were sampled from a fixed growthrate chemostat. In contrast, the average growth rate for the CG_mut population $\left(0.99 \pm 0.11 \mathrm{~h}^{-1}\right)$ increased and the standard deviation dropped in comparison to the CG strain $\left(0.87 \pm 0.19 \mathrm{~h}^{-1}\right)(p<0.0001)$ (Figure 4A), indicating a narrowing in the distribution of individual growth rates in the CG-mut population (Figure 4C). No significant difference was found in the lag time between the WT-mut population and the WT strain (Figure 4D,E). However, the mean lag time for the CG-mut cells was substantially shorter than that of the CG cells $(p<0.00001)$ (Figure 4D), since not only is there a high percentage of the CG-mut cells with a lag time $\leq 1.0 \mathrm{~h}$ but also no CG-mut cells showed a lag time $>1.5 \mathrm{~h}$ (Figure 4F). Clearly, the diversity of mutants that could persist in a chemostat is, in part, dependent on the genetic background of that parent strain.

Correlated Individual Growth Characteristics Revealed Changes in Heterogeneity. To further understand how genome reduction affects growth kinetics of the overall population, the individual $\mu$ and $\lambda$ values from these four populations were plotted in a scatter diagram (Figure 5). The diagrams reveal the heterogeneity of the populations, information that cannot be obtained from batch culture. If individual cells performed similarly, i.e., they were homogeneous, then all data spots would cluster due to similarity in both the $\mu$ and $\lambda$ values. In contrast, cellular heterogeneity is marked by an increased amount of scatter. These plots also provided clues as to which parameter drives the diversity of the population and whether there is dependency between these two parameters. Accordingly, individual growth characteristics for the CG strain were the most heterogeneous, with a broad scatter from both the $\mu$ and $\lambda$ parameters (Figure 5A). Interestingly, the CG_mut population were much more clustered compared with their parent strain, indicating that a far more homogeneous population of mutants survived in the chemostat environment (Figure 5B). The WT strain showed diversity in the $\mu$ parameter only; almost all the cells of that strain had no detectable lag time $\lambda$ (Figure 5C). The WT_mut population showed a slightly increased diversity in $\lambda$ and similar diversity in the $\mu$ parameter, thus the range of phenotypes that survived the chemostat was similar for the mutants (Figure 5D).

Cell Culturability of the Populations. A large number of nondividing cells were observed in all the populations under investigation and so a measure of culturability was investigated. Long-term observations indicated that these cells did not divide and were thus either quiescent or need a very long lag time $(>24 \mathrm{~h})$ to proliferate under the current nutrient condition (Supporting Information, Figure S3). Considering the purpose of this study, such nondividing cells were excluded from the above-mentioned growth kinetics assay.
We defined the culturability as the fraction of cells that grow, which was quantified by enumerating both dividing and nondividing cells. The culturability of the WT strain (62 \pm $11 \%)$ was similar to that of the derived WT_mut population $(63 \pm 11 \% ; p>0.05)$, both of which are three times higher than that of the CG strain $(20 \pm 6 \% ; p<0.005)$. The culturability of the CG_mut population $(45 \pm 2 \%)$ was twice as high as that of its parent CG strain $(p<0.05)$. It should be remembered that the chemostat selects for the fittest mutants and so this is to be expected. It is interesting that the WT_mut populations culturability is equal to that of the parent population and so the WT_mut strains' culturability has not been enhanced by the selection imposed by the chemostat.

\section{DISCUSSION}

The main motivations for producing genome-reduced strains of bacteria for synthetic biotechnologies are to alleviate protein burden and retain genome integrity. However, some reducedgenome strains have been reported to grow more slowly than their parental strains in batch culture, ${ }^{2,6}$ calling into question their efficiency. Genome integrity, or its effect on growth kinetics in a reduced genome strain has only recently been investigated, and it was found that in the context of metabolic stress, mutations accumulated quickly in these strains. The majority of these mutations ultimately led to less competitive phenotypes in chemostat populations. ${ }^{24}$ Therefore, it would be unlikely that most mutants would survive in the long chemostat run. Indeed, in a chemostat that is limited by a single resource, we would expect the single fittest, fastest growing, phenotype, mutant or otherwise, to "win out" and ultimately become monodominant. Yet even in clonal populations there appears to be variation in growth rates and hence phenotypes. What is not known is if that growth variability persists in the populations that remain in the harsh conditions in a chemostat. Thus, here, we have grown supposedly clonal populations of the WT strain (i.e., MG1655) and the derived reduced-genome CG strain (i.e., MDS42CG) in oligotrophic conditions in chemostats. We also grew mixed communities comprising solely of organisms that had acquired mutations in their $r p o B$ gene $\left(\right.$ rif $\left.^{\mathrm{R}}\right)$ or $c y c A$ gene $\left(\mathrm{cyc}^{\mathrm{R}}\right)$ for both strains. Each chemostat was sampled and the variability in the growth rates were characterized at the individual cell level using a facile microfluidic platform.

The Single-Cell Approach Enables Novel Measures of Population Heterogeneity. The facile microfluidic platform developed allowed us to observe cell growth in real time and quantify properties such as specific growth rate $\mu$ and lag time $\lambda$ of a population at the single-cell level. As illustrated in Figure 5, the direct correlation of $\mu$ and $\lambda$ of individual cells depicted key constitutional features of a population. Hidden information, such as overall heterogeneity of the growing cells and characteristics of each subset, are revealed at a glance. In combination with the culturability of a population, a clear relationship was shown where the culturability increased with the decrease of heterogeneity. For instance, the least culturable CG strain has the greatest degree of heterogeneity. It is apparent that these two parameters can serve as novel measures of robust growth. Notably, this crucial information, especially 
the individual $\mu-\lambda$ distributions, are inherently overlooked by conventional methods, therefore our method could contribute to more accurate understanding, modeling, and predicting of the bacterial responses to environmental changes.

The Effects of Genome Reduction on Cell Growth. We showed that average specific growth rates in the exponential growth phase obtained from on-chip culture are in excellent agreement with that from batch culture (Supporting Information, Figure S1 and Table 1), both of which show that the CG strain grow slower than the WT strain as reported previously. ${ }^{6}$ Importantly, with the ability of obtaining growth characteristics at single-cell resolution, our study provides the first measures of heterogeneity and culturability in these populations. We found that the genome-reduced CG strain not only had lower culturability than the WT strain but also had more heterogeneous growth characteristics than the parent WT strain. This observed increase in heterogeneity was present in both the growth rate and the lag time of individual cells. Given that one of the purposes of developing reduced genome chassis is to increase stability then this variability in growth might be unexpected. Furthermore, the microfluidics devices were loaded with cells sampled directly from the chemostats, where the organisms in the population were in a steady-state of growth. However, the variability might reflect the ability of individual microorganisms to respond to changes in local environment via modulations in gene expression, ${ }^{15,29-31}$ with more of the CG organisms being affected by the temporary stress of being transferred into the microfluidics device. Indeed, functional analyses of genome reductions in the CG strains show that they impacted global gene expression in these organisms by altering transcription of nondeleted regions of these genomes. ${ }^{6}$ This could be a crucial contributing factor to the growth heterogeneity observed in the CG strain.

Interestingly, our discoveries echo a recent study of the minimal genome organisms derived from Mycoplasma mycoides, which showed that as a greater number of genes are deleted from a genome, growth rates slow down. ${ }^{2}$ This trade-off is the result of genomes containing quasi-essential genes, which are thought to be coexpressed with the essential genes for robust growth. ${ }^{2}$ These genes could also be essential for increasing culturability of a strain, features that have not yet been investigated in any other reduced or minimal genomes strains. It is noteworthy that quasi-essential genes can be elusive, especially when comparative genomics are used as the main strategy for defining the minimal set of required genes. ${ }^{2}$ The 704 genes that were deleted to create the E. coli (MDS42) CG strains were targeted largely based on an in silico comparative genomics strategy to eliminate nonessential gene clusters. ${ }^{1}$ However, when strains bearing the systematic deletions were analyzed in depth for function, specifically growth properties in different environments, it was found that their impact was far more global than previously anticipated. ${ }^{6}$ Defects could not be attributed to single genes, instead clusters or genomic segments appeared to be involved. These segments impacted growth in specific environmental niches where they played a role in building new cellular material and conveying stress tolerance. In addition, these segments would have been acquired via horizontal gene transfer, thus comparative genomics, although a convenient strategy to define deep branching core genes, can miss recently acquired advantageous genomic changes that appear to be essential for good growth.

Mutations at Different Genetic Backgrounds. In a chemostat, one would expect the fastest growing organisms to be the most abundant, and ultimately, if all the organisms in the chemostat community are competing for the same limiting resource, the fittest organism should theoretically become monodominant. We grew up populations in chemostats that had acquired mutations through starvation and maintained the density at steady state. We know from a previous study ${ }^{24}$ that most starvation induced mutations have a deleterious effect on growth and thus we might expect a significant reduction in the diversity of growth characteristics in the mutants that survive and grow to dominate the chemostat. That is exactly what we found with the CG mut strains. Indeed, our microfluidic analysis showed that the average growth rate of cells sampled from the CG_mut population that grew to prominence in the chemostat exceeded that of the nonmutants (Table 1).

However, the effect of acquired mutations was less obvious in the WT_mut population; it had a similar average growth rate and retained the heterogeneity and culturablility of the parent WT strain. This diversity is perhaps surprising since both mutant populations are subject to the same stressful chemostat conditions. Thus, one might expect the chemostat to exert the same selection pressure on the WT_mut populations as for the CG_mut population and thus reduce the heterogeneity and increase cultureability; which does not seem to be the case. The fact that a wider range of growth characteristics are maintained and that no one phenotype wins out in the WT_mut population might suggest that growth is not entirely limited by the carbon substrate and that the complexity of the genome allows individuals to find or create niches in a way that the minimal genomes of the CG_muts cannot. Bearing in mind that CG strain was derived by deleting "nonessential" parts, including IS-elements, from the WT strain, and functional analyses of these deleted regions now show that they impact important phenotypes such as improved growth, stress tolerance and metabolic flexibility. ${ }^{6}$ It stands to reason that removing these sections could lead to organisms that are in general less phenotypically robust to mutations and thus a population where a few CG_muts have a clear advantage of others in way that does not occur with the WT muts. It has previously been shown that if mutations do occur in these two strain backgrounds, IS elements change their impact on the overall observed phenotype. ${ }^{32}$

The reduced genome chassis was conceived based on the hypothesis that the emergence of new traits, even if favorable, would be minimal in these systems. This is mainly to keep control of the process and product of the underlying biotechnology. ${ }^{33}$ However, spontaneous mutations do occur in populations over time, and so, their effect should be interrogated in these reduced genome strains. Our work here shows that in clonal populations of the reduced genome strains, the emerging mutations that are not deleterious and can survive in a selective chemostat environment are far more homogeneous than those that emerge in the wild type populations.

\section{CONCLUSION}

In this study, we developed a microfluidic system that enables fast quantification of growth rates and lag time of $E$. coli strains at the single-cell level. With this capability, we demonstrated the capacity to measure heterogeneity (from the correlated individual $\mu-\lambda$ distributions) and culturability of a population. We showed the increased culturability was associated with the decreased heterogeneity of a population for a reduced genome strain of E. coli grown in continuous culture in a chemostat. For a wild type strain and mutants that were derived through 
starvation stress, a surprising diversity of growth characteristics was retained in continuous culture populations. These discoveries illustrate that single-cell microfluidics can reveal subtle cellular responses that are obscure when observations are made at the population level.

\section{MATERIALS AND METHODS}

Fabrication of Microfluidic Platforms. Figure 1a shows the structure of the microfluidic platform designed for this study. There are two parallel $50(\mathrm{w}) \times 10(\mathrm{~h}) \mu \mathrm{m}$ channels, between which are eight identical $200(\mathrm{l}) \times 80(\mathrm{w}) \times 0.74(\mathrm{~h})$ $\mu \mathrm{m}$ microchambers. The submicron height of the chamber guaranteed cell capture and monolayer cell culture. Several circular pillars (diameter $=6 \mu \mathrm{m}$ ) are added to maintain the chamber height. To prevent cells from passing straight through the chambers during cell loading process (see below), a region with an even lower "head" height $(c a .0 .4 \mu \mathrm{m})$ was designed at the opposite side of the chamber (i.e., between the medium channel and main chambers).

Common soft lithography techniques were used to fabricate this structure. Briefly, SU8-2002 and SU8-3010 photoresist were diluted 1:4 and 1:1 respectively with cyclopentanone and then sequentially used to make features with differing heights, i.e., the low head-height region, microchambers, and two channels. After each photolithography/development step, the SU8 mold was hard baked at $180{ }^{\circ} \mathrm{C}$ for $15 \mathrm{~min}$ to avoid distortion in subsequent fabrication steps. The structure of the SU8 mold was measured using Bruker Dektak XT Height Profiler. When necessary, the fabrication process was optimized to meet the designed dimensions for each feature. After the silanization of the mold, polydimethylsiloxane (PDMS)/curing agent (Sylgard 184; Dow Corning Co., UK) (10/1) mixture was poured onto it and cured at $80{ }^{\circ} \mathrm{C}$ for overnight. Punching holes for both inlet and outlet, then the PDMS replica and clean coverslip were $\mathrm{O}_{2}$ plasma treated before bonding them together to make the microfluidic platform. The ca. $0.4 \mu \mathrm{m}$ height in parts of the device was verified using microscopic optical absorption measurements of a rhodamine solution, showing that these low height structures did not collapse (Supporting Information, Figure S4).

Bacterial Strains. All bacteria strains used in this study are derivatives of E. coli K12. The E. coli wide type strain, MG1655 (F- lambda- ilvG- rfb-50, rph-1) ${ }^{34}$ was purchased from the DSMZ culture collection (Braunsweig, Germany). The multiple deletion series (MDS) MDS42 strain has been described and characterized in detail previously, ${ }^{1}$ and was purchased from Scarab Genomics (Wisconsin, USA).

Chemostat Culture. For each growth experiment, precultures were always started from bacteria that had been cryopreserved at $-80{ }^{\circ} \mathrm{C}$ as glycerol stocks. Cells were first streaked onto LB agar plates and after overnight incubation at $37{ }^{\circ} \mathrm{C}$ a colony was transferred into $50 \mathrm{~mL}$ of $\mathrm{M} 9$ minimal medium that was supplemented with $10 \mathrm{mM}$ glucose. When this preculture had reached an $\mathrm{OD}_{600}$ of $0.1,8 \mathrm{~mL}$ of the culture was transferred to a computer-controlled glass bioreactor (modified miniBio 500, Applikon Biotechnology, Delft, NL myControl, Applikon Biotechnology Inc., CA) for continuous culture, with a working volume of $0.35 \mathrm{~L}$. The chemostat was fed M9 minimal medium supplemented with $1 \mathrm{mM}$ of glucose. Air passed into the reactor was via a $0.20 \mu \mathrm{m}$ filter. The impeller speed was set at $80 \mathrm{rpm}$, the temperature was $37^{\circ} \mathrm{C}$, and $\mathrm{pH}$ within the vessel was maintained at around 7.0. The medium-feed speed was set to maintain a dilution rate of 0.1 $\mathrm{h}^{-1}$. A steady state $\mathrm{OD}_{600}$ value of about 0.1 was reached approximately $20 \mathrm{~h}$ after this manipulation, at which point the cell cultures were ready for loading (after dilution, see below) into microchambers.

Generating Mutant Populations. To prepare mutant populations of the MG1655 and MDS42 strains, triplicate populations of each strain were maintained at steady state in glucose limited (M9 medium supplemented with $1 \mathrm{mM}$ glucose at a dilution rate of $0.1 \mathrm{~h}^{-1}$ ) chemostats for a duration of 21 days. During this time the cultures were assayed for spontaneously occurring mutations at either the $r p o B^{27}$ or the $c y c A^{28}$ loci by plating on selective solid media. A $1 \mathrm{~mL}$ of subsample from each chemostat was plated and monitored for the number of colonies resistant to the antibiotics Rifampicin or D-Cycloserine. Mutation accumulation data from the chemostat experiments were surveyed to find a population from each strain with a similar mutant fraction. For the MG1655 strain, mutants were sampled from a population with an estimated total of 34660 mutants $/ \mathrm{mL}\left(2.8 \times 10^{-2} \%\right)$. Similarly, for the MDS42 strain, mutants were chosen from a population with an estimate of 38,108 mutants $/ \mathrm{mL}\left(2.2 \times 10^{-2} \%\right)$. Both populations had been in continuous, glucose-limited culture for 14 days, and had thus gone through an equivalent number of generations (N.B.: generation time is fixed in a chemostat). At the time of sampling, up to 140 resistant colonies were picked and stored as glycerol stocks at $-80{ }^{\circ} \mathrm{C}$. These were recovered in $\mathrm{LB}$ medium and pooled to make the WT mut (mutants derived from a MG1655 chemostat population) and the CG_mut (mutants derived from a MDS42 chemostat population). Before inoculating the microfluidics device, a $1 \mathrm{~mL}$ subculture of each population was used to start up a chemostat culture, which grew using the conditions described below.

On-Chip Bacteria Culture. The microfluidic platform was initially filled with M9 minimal medium containing $10 \mu \mathrm{M}$ glucose, which was delivered through the two parallel channels at $10 \mu \mathrm{L} / \mathrm{min}$ with a syringe pump (NE-1000, New Era Pump Systems Inc.) (Figure 1). Prior to introducing to the microfluidic chip, bacterial culture from the chemostat was diluted 10 - or 20 -fold with glucose-containing fresh medium. After twice centrifuging at $3000 \mathrm{rpm} \times 5 \mathrm{~min}$ and resuspending in the same fresh medium, the sample was loaded into the device through the "loading" channel. The time was ca. $30 \mathrm{~min}$ between subsampling from the chemostat and introducing to the microfluidic chip. After loading the cells into the device, the same fresh medium was allowed to flow through either side of the chambers at a suitable flow rate to remove nontrapped cells in both channels. Meanwhile cell density of the microchambers could be adjusted by changing the flow rate. Thereafter, the flow rate was changed to $0.1 \mu \mathrm{L} / \mathrm{min}$ to start on-chip cell culture. This continuous flow culture provided constant nutrient supply to the cells and simultaneously eliminated metabolic waste. The whole microfluidic platform was mounted into a commercial temperature-controlled chamber (Heating Insert P Lab-Tek 2000, PeCon GmbH, Germany) (Figure 1B). It was preheated to $37{ }^{\circ} \mathrm{C}$ and mounted on motorized microscope stage. Simple calculations show that at the flow rates used, the medium reaches $37^{\circ} \mathrm{C}$ a few seconds after being driven into the platform. Unless denoted, the on-chip cell culture unusually lasted for $\sim 6 \mathrm{~h}$ before the chamber was fully filled with bacteria, which affects clear discrimination of the boundaries between the colonies.

Optical Image Acquisition and Analysis. Time-lapse images were recorded for five or $6 \mathrm{~h}$ with an IX71 epi- 
fluorescence inverted microscope (Olympus Inc., UK), equipped with a long working distance Olympus LCP FL $100 \times / 0.85$ objective lens, an Andor iXon Ultra 897 CCD camera, and a motorized stage (SCAN IM $120 \times 80-2 \mathrm{~mm}$, Marzhauser Wetalar GmbH\&Co.KG, Germany). The whole process was automatically controlled through the interface of the open source software Micro-Manager. The high magnification of the objective lens enabled the bacteria images clear enough for quantitative image analysis. To calculate the total area of a single colony starting from a single cell, whereby to obtain its growth rate $(\mu)$ and lag time $(\lambda)$ parameters (see below), all the time-lapse images were processed with ImageJ (Supporting Information, Figure S2), as detailed previously. ${ }^{19}$ With the chosen microscope focus setting, the bacteria typically appeared as bright objects surrounded by a dark rim (Figure 2), which made it easy to identify and calculate colony area.

Calculation of $\boldsymbol{\mu}$ and $\lambda$ Parameters. For traditional batch culture, cell growth rates are defined as those measured during the exponential growth phase. The specific growth rate $(\mu)$ can be derived from optical density measurements using eq $1^{35}$

$$
\mu=\ln \left(\mathrm{OD}_{t} / \mathrm{OD}_{0}\right) / T
$$

where $\mathrm{OD}_{0}$ and $\mathrm{OD}_{t}$ are the optical density (OD) of suspended cells (i.e., an indirect measure of cell density as mass per unit volume), $T$ is the length of time for this change.

In our microfluidic platforms, the bacteria grow as monolayer microcolonies starting from a single cell, so the cell mass can be considered to be proportional to the colony's area $(S)$. Therefore, the growth rate of bacteria within the colony was analyzed via the following equation ${ }^{19,20}$

$$
\mu=\ln \left(S_{t} / S_{0}\right) /(t-\lambda)
$$

where $S_{0}$ and $S_{t}$ are the areas of a single colony at the initial time $(t=0)$ and at time $t$, and $\lambda$ is the lag time, which is defined as the time taken for the initial cell division to occur. ${ }^{36}$ This is close to the point of intersection of the steepest slope of the growth curve with the time-axis (Supporting Information, Figure S2). ${ }^{13,35,37}$

Statistics. A total of 80 randomly selected single cells colonies from three independent experiments were analyzed for each population so as to allow the derivation of statistically meaningful characteristics. ${ }^{20,38}$ Unless noted, average values and standard deviation from the triplicate are given. All statistical analysis was performed using Prism software. A difference was regarded as significant when $p<0.05(*), p<0.01(* *), p<$ $0.001(* * *)$ and $p<0.0001$ (****) following a two-way ANOVA with post hoc testing unless otherwise denoted.

\section{ASSOCIATED CONTENT}

\section{S Supporting Information}

The Supporting Information is available free of charge on the ACS Publications website at DOI: 10.1021/acssynbio.7b00177.

Figure S1, Growth rates of MG1655 and MDS42 strains in batch culture; Figure S2, Image analysis and calculation of specific growth rate $(\mu)$ and lag time $(\lambda)$ of single cells; Figure S3, Long-term culture of cells in the microfluidic device; Figure S4, Determination of PDMS chamber height using microscopic absorbanceconcentration measurements (PDF)

\section{AUTHOR INFORMATION}

\section{Corresponding Authors}

*E-mail: william.sloan@glasgow.ac.uk.

*E-mail: huabing.yin@glasgow.ac.uk.

ORCID $\odot$

Xiaofei Yuan: 0000-0001-5395-9109

Huabing Yin: 0000-0001-7693-377X

Notes

The authors declare no competing financial interest.

\section{ACKNOWLEDGMENTS}

This work was supported by Frontier Engineering-Early Stage Researcher Catalyst Funding (EPSRC EP/K038885/1). Portions of this work were performed in the James Watt Nanofabrication Centre (JWNC) in the University of Glasgow.

\section{REFERENCES}

(1) Posfai, G., Plunkett, G., Feher, T., Frisch, D., Keil, G. M. Umenhoffer, K., Kolisnychenko, V., Stahl, B., Sharma, S. S., de Arruda, M., Burland, V., Harcum, S. W., and Blattner, F. R. (2006) Emergent properties of reduced-genome Escherichia coli. Science 312, 10441046.

(2) Hutchison, C. A., Chuang, R. Y., Noskov, V. N., Assad-Garcia, N., Deerinck, T. J., Ellisman, M. H., Gill, J., Kannan, K., Karas, B. J., Ma, L., Pelletier, J. F., Qi, Z. Q., Richter, R. A., Strychalski, E. A., Sun, L., Suzuki, Y., Tsvetanova, B., Wise, K. S., Smith, H. O., Glass, J. I., Merryman, C., Gibson, D. G., and Venter, J. C. (2016) Design and synthesis of a minimal bacterial genome. Science 351, aad6253.

(3) Li, B., Qiu, Y., Shi, H., and Yin, H. (2016) The importance of lag time extension in determining bacterial resistance to antibiotics. Analyst 141, 3059-3067.

(4) Nikel, P. I., Chavarria, M., Danchin, A., and de Lorenzo, V. (2016) From dirt to industrial applications: Pseudomonas putida as a Synthetic Biology chassis for hosting harsh biochemical reactions. Curr. Opin. Chem. Biol. 34, 20-29.

(5) Lane, N., and Martin, W. (2010) The energetics of genome complexity. Nature 467, 929-934.

(6) Karcagi, I., Draskovits, G., Umenhoffer, K., Fekete, G., Kovacs, K., Mehi, O., Baliko, G., Szappanos, B., Gyorfy, Z., Feher, T., Bogos, B., Blattner, F. R., Pal, C., Posfai, G., and Papp, B. (2016) Indispensability of horizontally transferred genes and its impact on bacterial genome streamlining. Mol. Biol. Evol. 33, 1257-1269.

(7) Shimizu, Y., Tsuru, S., Ito, Y., Ying, B. W., and Yomo, T. (2011) Stochastic switching induced adaptation in a starved Escherichia coli population. PLoS One 6, e23953.

(8) Kaern, M., Elston, T. C., Blake, W. J., and Collins, J. J. (2005) Stochasticity in gene expression: from theories to phenotypes. Nat. Rev. Genet. 6, 451-464.

(9) Elowitz, M. B., Levine, A. J., Siggia, E. D., and Swain, P. S. (2002) Stochastic gene expression in a single cell. Science 297, 1183-1186.

(10) Fraser, C., Hanage, W. P., and Spratt, B. G. (2007) Recombination and the nature of bacterial speciation. Science 315, 476-480.

(11) Probst, C., Grünberger, A., Wiechert, W., and Kohlheyer, D. (2013) Polydimethylsiloxane (PDMS) sub-micron traps for single-cell analysis of bacteria. Micromachines 4, 357-369.

(12) Choi, J., Jung, Y. G., Kim, J., Kim, S., Jung, Y., Na, H., and Kwon, S. (2013) Rapid antibiotic susceptibility testing by tracking single cell growth in a microfluidic agarose channel system. Lab Chip 13, 280287.

(13) Peitz, I., and van Leeuwen, R. (2010) Single-cell bacteria growth monitoring by automated DEP-facilitated image analysis. Lab Chip 10, 2944-2951.

(14) Elfwing, A., LeMarc, Y., Baranyi, J., and Ballagi, A. (2004) Observing growth and dof large numbers of individual bacteria by image analysis. Appl. Environ. Microbiol. 70, 675-678. 
(15) Wang, P., Robert, L., Pelletier, J., Dang, W. L., Taddei, F., Wright, A., and Jun, S. (2010) Robust growth of Escherichia coli. Curr. Biol. 20, 1099-1103.

(16) Long, Z., Olliver, A., Brambilla, E., Sclavi, B., Lagomarsino, M. C., and Dorfman, K. D. (2014) Measuring bacterial adaptation dynamics at the single-cell level using a microfluidic chemostat and time-lapse fluorescence microscopy. Analyst 139, 5254-5262.

(17) Hou, Z., An, Y., Hjort, K., Hjort, K., Sandegren, L., and Wu, Z. (2014) Time lapse investigation of antibiotic susceptibility using a microfluidic linear gradient 3D culture device. Lab Chip 14, 34093418.

(18) Cattoni, D. I., Fiche, J. B., Valeri, A., Mignot, T., and Nollmann, M. (2013) Super-resolution imaging of bacteria in a microfluidics device. PLoS One 8, e76268.

(19) Li, B., Qiu, Y., Glidle, A., Mcllvenna, D., Luo, Q., Cooper, J., Shi, H. C., and Yin, H. (2014) Gradient microfluidics enables rapid bacterial growth inhibition testing. Anal. Chem. 86, 3131-3137.

(20) Li, B., Qiu, Y., Glidle, A., Cooper, J., Shi, H., and Yin, H. (2014) Single cell growth rate and morphological dynamics revealing an "opportunistic" persistence. Analyst 139, 3305-3313.

(21) Akeno, Y., Ying, B.-W., Tsuru, S., and Yomo, T. (2014) A reduced genome decreases the host carrying capacity for foreign DNA. Microb. Cell Fact. 13, 49-57.

(22) Csorgo, B., Feher, T., Timar, E., Blattner, F. R., and Posfai, G. (2012) Low-mutation-rate, reduced-genome Escherichia coli: an improved host for faithful maintenance of engineered genetic constructs. Microb. Cell Fact. 11, 11-23.

(23) Ying, B.-W., Seno, S., Kaneko, F., Matsuda, H., and Yomo, T. (2013) Multilevel comparative analysis of the contributions of genome reduction and heat shock to the Escherichia coli transcriptome. BMC Genomics 14, 25-37.

(24) Couto, J. M., McGarrity, A., Russell, J., and Sloan, W. T. The effect of metabolic stress on genome stability of a synthetic biology chassis Escherichia coli K12 strain (in review).

(25) Ferenci, T. (2008) Bacterial physiology, regulation and mutational adaptation in a chemostat environment. Adv. Microb. Physiol. 53, 169-229.

(26) Sambrook, J., and Russell, D. W. (2001) Molecular Cloning-A Laboratory Manual, 3rd ed., Cold Spring Harbor, New York.

(27) Garibyan, L., Huang, T., Kim, M., Wolff, E., Nguyen, A., Nguyen, T., Diep, A., Hu, K., Iverson, A., Yang, H., and Miller, J. H. (2003) Use of the rpoB gene to determine the specificity of base substitution mutations on the Escherichia coli chromosome. DNA Repair 2, 593-608.

(28) Feher, T., Cseh, B., Umenhoffer, K., Karcagi, I., and Posfai, G. (2006) Characterization of cycA mutants of Escherichia coli. An assay for measuring in vivo mutation rates. Mutat. Res., Fundam. Mol. Mech. Mutagen. 595, 184-190.

(29) Soupene, E., van Heeswijk, W. C., Plumbridge, J., Stewart, V., Bertenthal, D., Lee, H., Prasad, G., Paliy, O., Charernnoppakul, P., and Kustu, S. (2003) Physiological studies of Escherichia coli strain MG1655: Growth defects and apparent cross-regulation of gene expression. J. Bacteriol. 185, 5611-5626.

(30) King, T., Kocharunchitt, C., Gobius, K., Bowman, J. P., and Ross, T. (2014) Global genome response of Escherichia coli O157ratioH7 Sakai during dynamic changes in growth kinetics induced by an abrupt temperature downshift. PLoS One 9, e99627.

(31) Matsumoto, Y., Murakami, Y., Tsuru, S., Ying, B.-W., and Yomo, T. (2013) Growth rate-coordinated transcriptome reorganization in bacteria. BMC Genomics 14, 808-817.

(32) Umenhoffer, K., Feher, T., Baliko, G., Ayaydin, F., Posfai, J., Blattner, F. R., and Posfai, G. (2010) Reduced evolvability of Escherichia coli MDS42, an IS-less cellular chassis for molecular and synthetic biology applications. Microb. Cell Fact. 9, 38.

(33) Editorial, N. B. (2009) Unbottling the genes. Nat. Biotechnol. 27, 1059.

(34) Blattner, F. R., Plunkett, G., Bloch, C. A., Perna, N. T., Burland, V., Riley, M., Collado-Vides, J., Glasner, J. D., Rode, C. K., Mayhew, G. F., Gregor, J., Davis, N. W., Kirkpatrick, H. A., Goeden, M. A., Rose, D.
J., Mau, B., and Shao, Y. (1997) The complete genome sequence of escherichia coli K-12. Science 277, 1453-1462.

(35) Hall, B. G., Acar, H., Nandipati, A., and Barlow, M. (2014) Growth rates made easy. Mol. Biol. Evol. 31, 232-238.

(36) Pirt, S. J. (1975) Principles of Microbe and Cell Cultivation, Wiley.

(37) Smelt, J. P. P. M., Otten, G. D., and Bos, A. P. (2002) Modelling the effect of sublethal injury on the distribution of the lag times of individual cells of Lactobacillus plantarum. Int. J. Food Microbiol. 73, 207-212.

(38) Yin, H., Zhang, X., Pattrick, N., Klauke, N., Cordingley, H. C., Haswell, S. J., and Cooper, J. M. (2007) Influence of hydrodynamic conditions on quantitative cellular assays in microfluidic systems. Anal. Chem. 79, 7139-7144. 